\title{
NOTES ON CERTAIN INEQUALITIES BY HÖLDER, LEWENT AND KY FAN
}

\author{
Milan V. Jovanović, Tibor K. PoGány And JózSEF SÁndor
}

\begin{abstract}
The aim of this paper is twofold. First we show that the famous Hölder inequality (which one, according to [10] should be called as the Rogers inequality) was discovered by Grolous [5] and Besso [3] about 10 years before Rogers. On the other hand, a result obtained by Lewent [9] leads to a new proof of the famous Ky Fan inequality [2]. Related results are pointed out, too.
\end{abstract}

Mathematics subject classification (2000): 01A85, 01A55, 01A70, 26D15.

Key words and phrases: History of inequalities, Hölder's, Jensen's, Ky Fan's inequalities.

\section{REFERENCES}

[1] H. AlZER, The inequality of Ky Fan and related results, Acta Appl. Math. 38 (1995), 305-354.

[2] E. F. BeckenBach And R. Bellman, Inequalities, Springer-Verlag, Berlin, New York, Tokyo, 1961.

[3] D. Besso, Teoremi elementari sui massimi e minimi, Annuario de Ist. Tecnico di Roma (1879); Boll. di Mat. 8 (1907), 57-74.

[4] V. Govedarica AND M. Jovanović, On the inequalities of Ky Fan, Wang-Wang and Alzer, J. Math. Anal. Appl. 270 (2002), 709-712.

[5] J. GROLOUS, Un théorème sur les fonctions, L'Institut, Journal Universel des Sciences et des Sociétés Savantes en France et a l'Étranger (2) III (1875), 401.

[6] G. H. Hardy, J. E. LitTlewood AND G. Pólya, Inequalities (2nd ed.) Cambridge University Press, London, New York, 1952.

[7] O. HöLDER, Über einen Mittelwertssatz, Nachr. König. Ges. Wiss. und GA Univ. Göttingen 2 (1889), 38-47.

[8] J. L. W. V. JENSEN, Sur les fonctions convexes et les inégalités entre les valeurs moyennes, Acta Math. 30 (1906), 175-193 ; Nyt. Tidsskr. Math. 16B (1905), 49-68.

[9] L. LEWENT, Über einige Ungleichungen, Sitzungsber. Berl. Math. Ges. 7 (1908), 95-100.

[10] L. MALIGRANDA, Why Hölder's inequality should be called Rogers's inequality, Math. Ineq. Appl. 1(1) (1998), 69-83.

[11] D. S. Mitrinović, J. E. PeČARIĆ And A. M. Fink, Classical and New Inequalities in Analysis, Kluwer Academic Publisher, Dordrecht, 1993.

[12] E. Neuman AND J. SÁNDOR, On the Ky Fan inequality and related inequalities I, Math. Ineq. Appl. 5 (2002), 49-56; II, Bull. Austral. Math. Soc. 72(2005), 87-107.

[13] J. SÁNDOR, On an inequality of Ky Fan, Preprint 90-7, Babeş-Bolyai Univ., 29-34; II, Intern. J. Math. Ed. Sci. Tech. 22 (1991), 326-328; III, Intern. J. Math. Ed. Sci. Tech., 32 (2001), 133-160.

[14] J. SÁNDOR AND T. TRIF, A new refinement of the Ky Fan inequality, Math. Ineq. Appl. 2 (1999), 529-533.

[15] F. SiBIRANI, Intorno alle funzioni convesse, Lomb. Ist. Rend. (2)40 (1907), 903-919.

[16] www.emis.de -- JFM 40.0337.04 